\title{
Large Epitheloid Vulvar Angiosarcoma in Post Menopausl Woman; a Rare Case Report \\ Dr. Priyanka Das ${ }^{1 *}$, Dr. Ramesh Sonowal ${ }^{2}$
}

\author{
${ }^{1}$ Senior Resident, Assam Medical College and Hospital, Dibrugarh, Assam-786002, India \\ ${ }^{2}$ Professor, Head of Department, Obst and Gynae Department, Assam Medical College and Hospital, Dibrugarh, Assam-786002, India
}

DOI: $\underline{10.36347 / \mathrm{sasjs} .2021 . v 07 i 03.007}$

| Received: 12.02.2021 | Accepted: 17.03.2021 | Published: 21.03.2021

*Corresponding author: Dr. Priyanka Das

\section{Abstract}

Angiosarcoma is a rare neoplasm typically presenting in the skin and superficial soft tissues which comprise less than $2 \%$ of all sarcomas and are aggressive in nature. They often present with a bruise-like appearance which can progress to nodular and ulcerated lesions. While angiosarcoma can arise anywhere in the body, they rarely occur in the female genital tract. This case report of a 40 years old post menopausal women attending the Gynae OPD of Assam Medical College with a swelling in the left side of the vulva for last 2 years which was progressively increasing in size. It was painless causing discomfort while walking. There was no history of discolouration of skin, prior radiation therapy, family history, difficulty in micturition or any discharge per vaginum. Her bowel habit was normal. Her general condition was fair and vitals were stable. Local examination revealed a $(10 \times 10 \times 4) \mathrm{cm}$ lump extending from the mons pubis to the left labia majora without inflammatory signs or discharge. Clitoris and rest of the vulva was normal but the urethral meatus was deviated to the right side. Examination of the vagina revealed a left sided large swelling with normal introitus and pelvis was normal and there was no inguinal lymphadenopathy. The patient was planned for surgery and enucleation was done. Histopathology revealed it to be an epithelioid vulvar angiosarcoma. The literature supports surgical excision as the primary treatment of choice for angiosarcoma, although the prognosis remains poor even with early intervention whereas loco- regional and systemic chemotherapy have shown little success in improving survival. Given the overall poor prognosis of angiosarcoma, early detection remains the key element in maximizing patient outcome.

Keywords: Epithelioid angiosarcoma, postmenopausal.

Copyright $(\mathcal{C} 2021$ The Author(s): This is an open-access article distributed under the terms of the Creative Commons Attribution 4.0 International License (CC BY-NC 4.0) which permits unrestricted use, distribution, and reproduction in any medium for non-commercial use provided the original author and source are credited.

\section{INTRODUCTION}

Angiosarcoma is a rare malignancy which typically presents in the skin and superficial soft tissues. Angiosarcomas comprise less than $2 \%$ of all sarcomas and they are commonly aggressive in nature [1, 2]. They often present as bruise-like which can progress to nodular and ulcerated lesions. While angiosarcomas can arise anywhere in the body, they rarely occur in the female genital tract [3].

Primary angiosarcomas arise without recognized associated risk factors [2]. Certain familial syndromes such as Li Fraumeni and neurofibromatosis, as well as environmental factors such as chronic inflammation, and foreign body material are associated with development of secondary angiosarcomas [1-4]. Chronic lymph edema and radiation treatment are also known risk factors for development of angiosarcoma, which has been well described primarily in patients treated for breast cancer [5]. Lymphangiosarcoma occurring secondary to chronic lymph edema in patients who had undergone mastectomy was first reported in 1948 and is now known as Stewart-Treves syndrome [6]. This unique case report describes primary case of large epitheloid vulvar angiosarcoma in labia majora of a post menopausal woman.

\section{CASE REPORT}

A 40 year old Para $_{3}$ attended the Gynae OPD of Assam Medical College with a painless mass in the left side of the vulva. The mass was large enough to cause discomfort in walking. There was no history of vulvar rash, trauma, prior radiation therapy, family history, difficulty in micturition or swelling of limbs. She attained menopause two year back. Her bowel habit was normal. Her general condition was fair and vitals were stable. Local examination revealed a $(10 \times 10 \times 4)$ $\mathrm{cm}$ lump extending from the mons pubis to the left labia majora without inflammatory signs or discharge. Clitoris and rest of the vulva was normal but the urethral meatus was deviated to the right side. 
Priyanka Das \& Ramesh Sonowal., SAS J Surg, Mar, 2021; 7(3): 137-140

Examination of the vagina revealed a left sided large swelling with normal introitus and pelvis was normal and there was no inguinal lymphadenopathy. The patient was planned for surgery. Incision was given over the anterior surface of the tumor and enucleation was done. Histopathology and immunohistochemistry was done in Pathology department of Assam Medical
College which revealed epithelioid angiosarcoma. Neoplastic cells were positive for CD31, Vimentin and EMA whereas negative for Desmin, S100, Myogenin, SMA. Ethical clearance was taken from Institutional Ethics Committee $(\mathrm{H})$ of Assam Medical College and Hospital, Dibrugarh.
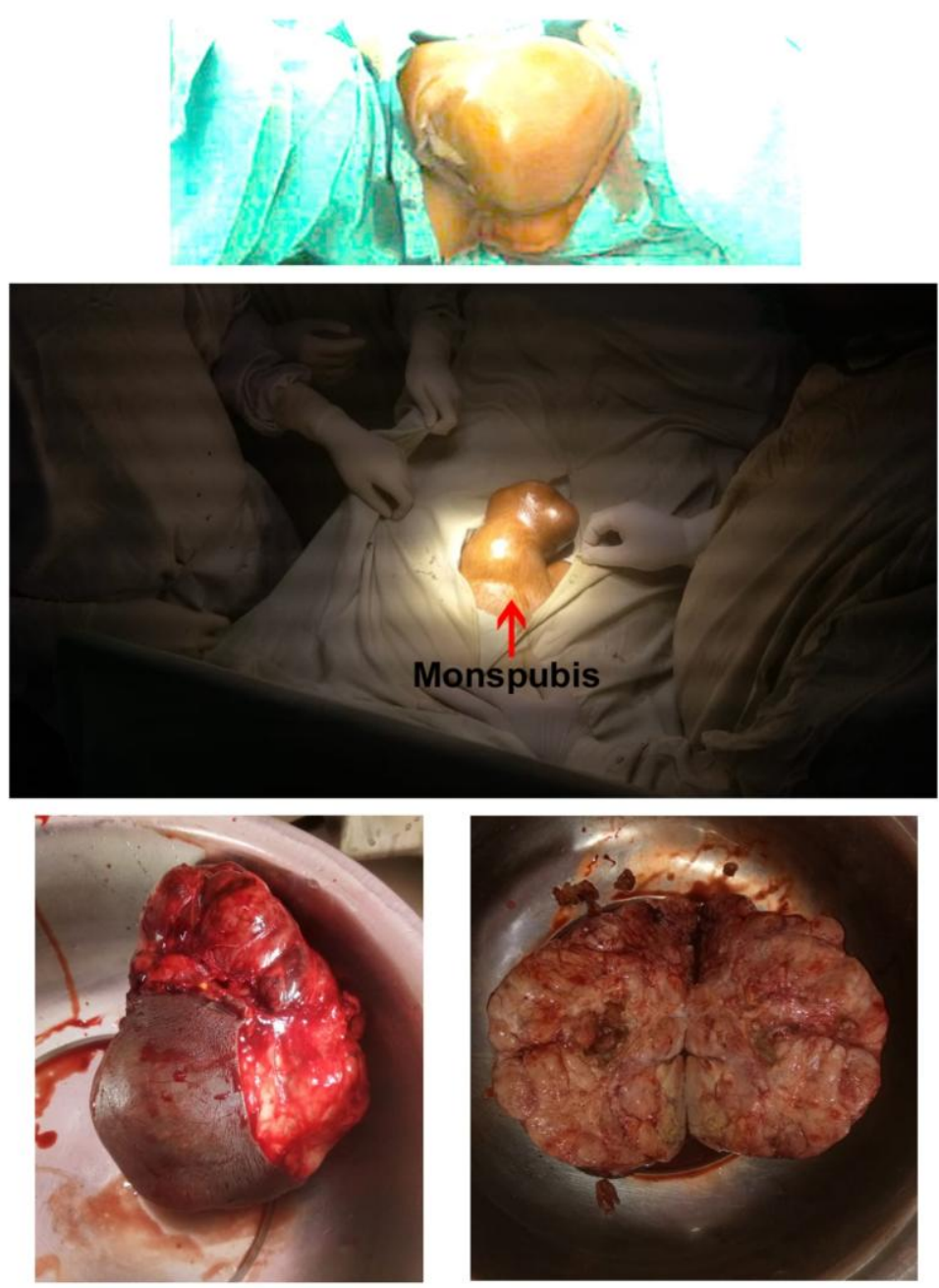

Immunohistochemistry

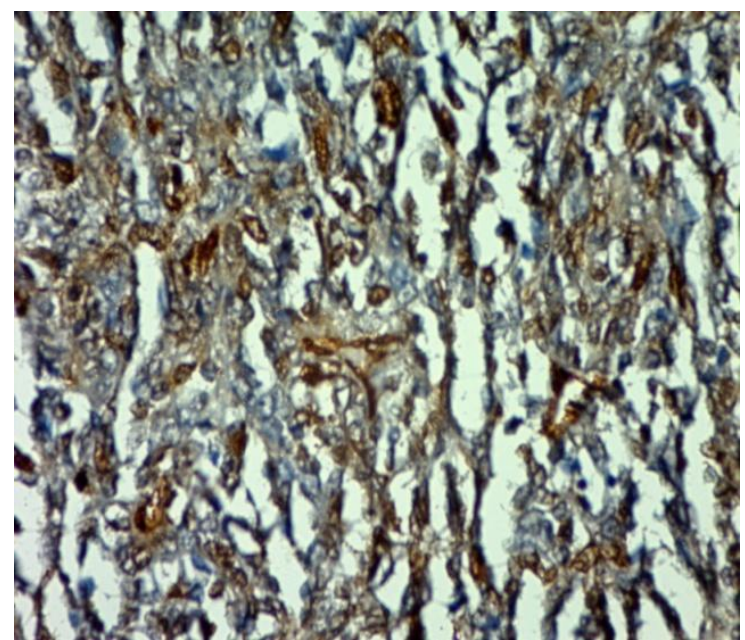

Immunohistochemical staining at $10 \times$ : A) CD31

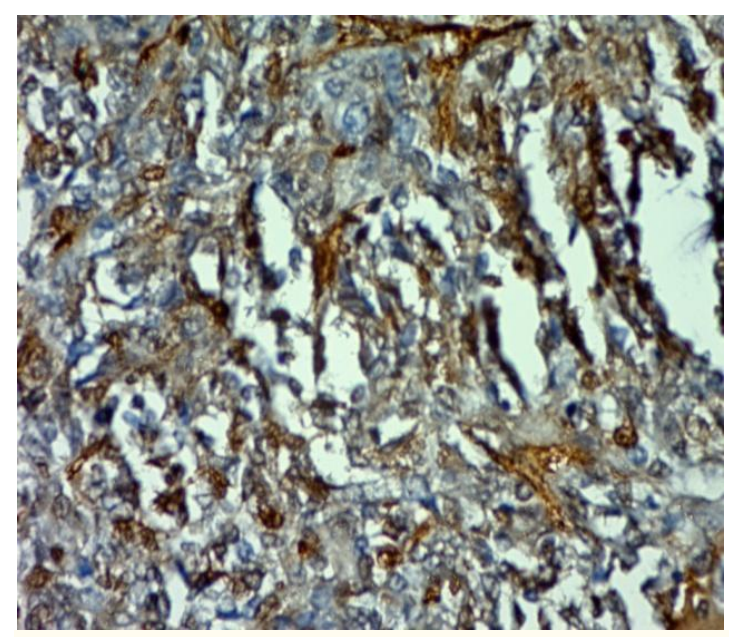

Immunohistochemical staining at $40 \times$ : B) CD31 
Priyanka Das \& Ramesh Sonowal., SAS J Surg, Mar, 2021; 7(3): 137-140
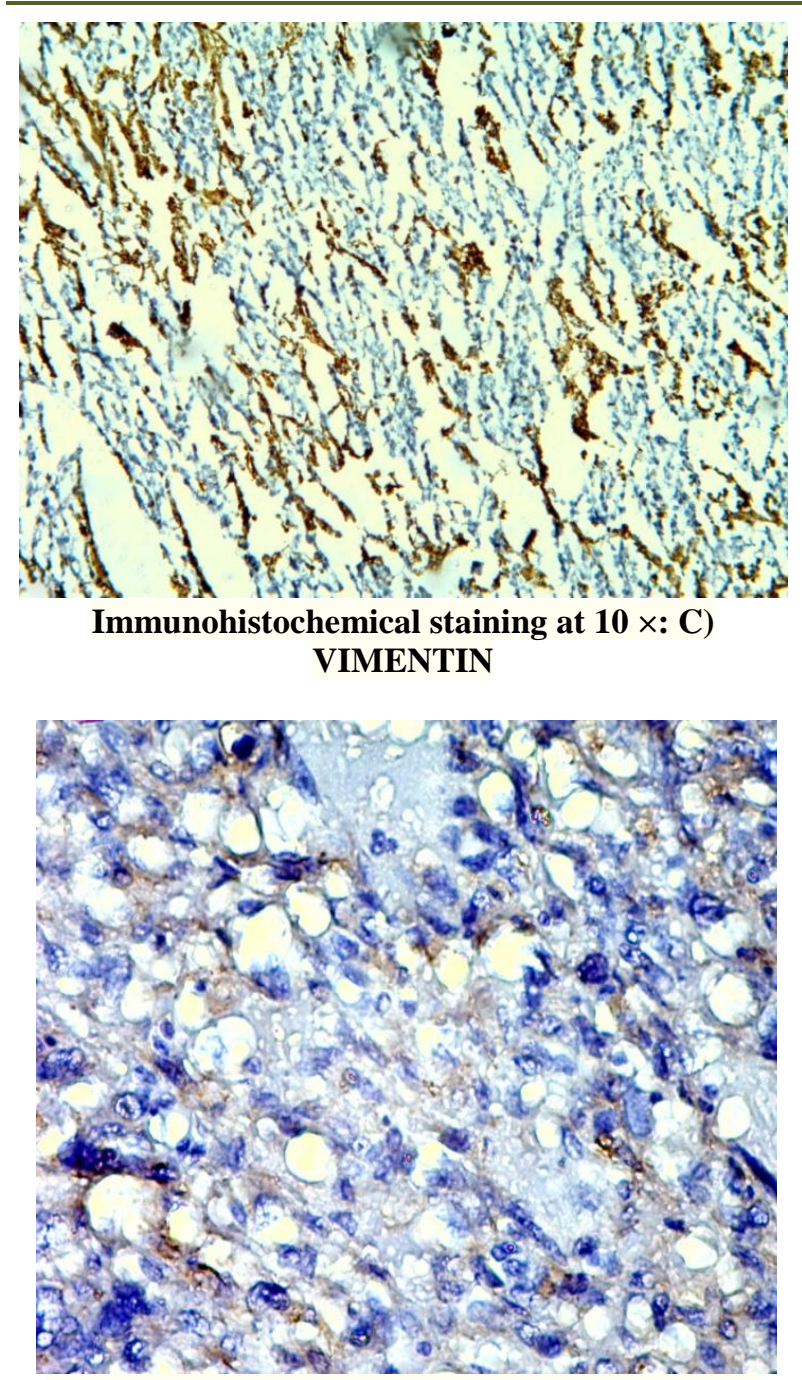

Immunohistochemical staining at $10 \times$ : D) EMA

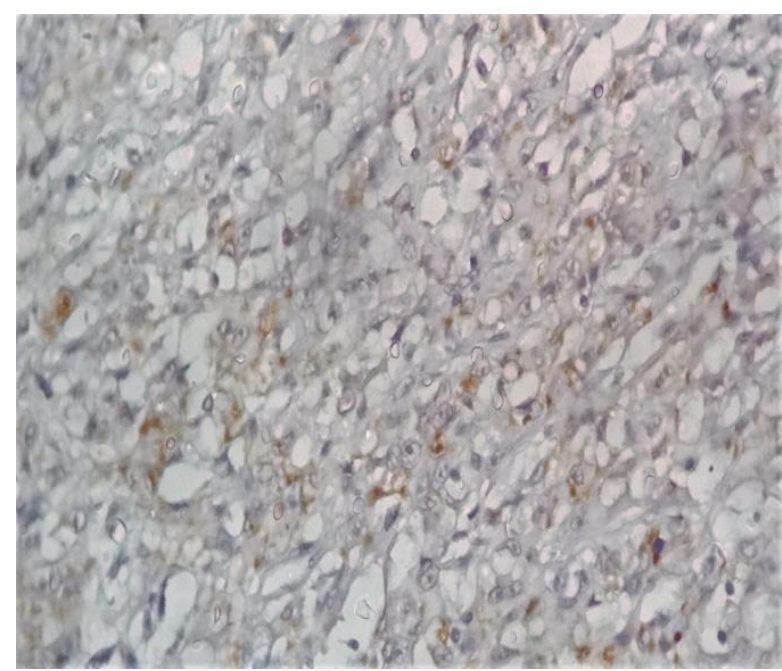

Immunohistochemical staining at $10 \times$ : E) CD31

\section{DISCUSSION}

Clinical diagnosis of angiosarcoma may be delayed due to the asymptomatic nature and difficulty in differentiating between trauma, infection or malignancy. Cutaneous angiosarcoma in general typically present as a violaceous papule/plaque or a bruise-like appearance while deeper lesions typically present as an expanding painless mass as seen in our patient [7]. This non-specific presentation can lead to delays in diagnosis, particularly when the patient is not experiencing any discomfort from the lesion.

The epithelioid subtype seen in our patient is so diagnosed because unlike other angiosarcoma the morphology resembles epithelial tissue, and thus can be easily mistaken for a carcinoma. Immunohistochemical staining can help differentiate epithelioid angiosarcoma from carcinoma as well as other epithelioid neoplasm.

The literature supports surgical excision as the primary treatment of choice for angiosarcoma, although the prognosis remains poor even with early intervention [8]. Loco regional and systemic chemotherapy have shown little success in improving survival [7]. Among the vulvar angiosarcoma cases in the literature, treatment approaches have included surgical excision in all cases with adjuvant radiotherapy and/or chemotherapy in most [9-11].

Due to the rarity of angiosarcoma, information on prognosis is limited. Epithelioid angiosarcoma tend to metastasize early both to solid organs (especially lungs, bone, soft tissue, skin) and lymph nodes [12]. The overall survival rate for angiosarcoma at 5 years is approximately $35 \%$ [7]. The epithelioid subtype is rare enough that no data is available to compare survival to other subtypes. High proliferative index (MIB-1 at least $10 \%$ ), older age, and larger tumor size are poor prognostic factors [12].

\section{CONCLUSION}

Given the overall poor prognosis of angiosarcoma, early detection remains the key element in maximizing patient outcome. Further complicating the diagnosis is the varied clinical presentation; while our patient presented with painless masses the other cases of vulvar angiosarcoma described presents as violaceous skin, or white papules. Thus, for any suspicious lesion of the vulva, a biopsy should be considered, particularly if it occurs in a previously radiated field or an area of chronic lymph edema which are the risk factors for angiosarcoma. Given the long asymptomatic duration generally seen, educating the patient on the importance of resorting to the physician if any lesions develop would be appropriate given that many patients will be outside the window of regular check up.

\section{REFERENCES}

1. Koch M, Nielsen GP, Yoon SS. Malignant tumors of blood vessels: angiosarcomas, hemangioendotheliomas, and hemangioperictyomas. Journal of surgical oncology. 2008 Mar 15;97(4):321-9.

2. Guirguis A, Kanbour-Shakir A, Kelley J. Epithelioid angiosarcoma of the mons after chemoradiation for vulvar cancer. International 
journal of gynecological pathology. $2007 \mathrm{Jul}$ 1;26(3):265-8.

3. Young RJ. Angiosarcoma 1. Lancet Onco. 2010 Oct; 11(10):983-991.

4. Jennings TA, Peterson L, Axiotis CA, Friedlaender GE, Cooke RA, Rosai J. Angiosarcoma associated with foreign body material: a report of three cases. Cancer. 1988 Dec 1;62(11):2436-44.

5. Huang J, Mackillop WJ. Increased risk of soft tissue sarcoma after radiotherapy in women with breast carcinoma. Cancer. 2001 Jul 1;92(1):17280 .

6. Stewart FW, Treves N. Lymphangiosarcoma in postmastectomy lymphedema. A report of six cases in elephantiasis chirurgica. Cancer. 1948 May;1(1):64-81.

7. Young RJ, Brown NJ, Reed MW, Hughes D, Woll PJ. Angiosarcoma. The lancet oncology. 2010 Oct 1;11(10):983-91.

8. Sharma A, Schwartz RA. Stewart-Treves syndrome: pathogenesis and management. Journal of the American Academy of Dermatology. 2012 Dec 1;67(6):1342-8.

9. Kruse AJ, Sep S, Slangen BF, Vandevijver NM, Van Gorp T, Kruitwagen RF, Van de Vijver KK. Angiosarcomas of primary gynecologic origin: a clinicopathologic review and quantitative analysis of survival. International Journal of Gynecologic Cancer. 2014 Jan 1;24(1):4-12.

10. Guirguis A, Kanbour-Shakir A, Kelley J. Epithelioid angiosarcoma of the mons after chemoradiation for vulvar cancer. International journal of gynecological pathology. $2007 \mathrm{Jul}$ 1;26(3):265-8.

11. Sheinis M, Cesari M, Selk A. Angiosarcoma of the vulva: a case report. Journal of lower genital tract disease. 2016 Jan 1;20(1):e6-7.

12. Hart J, Mandavilli S. Epithelioid angiosarcoma: a brief diagnostic review and differential diagnosis. Archives of pathology \& laboratory medicine. $2011 \mathrm{Feb} ; 135(2): 268-72$. 